\title{
Investigación formativa: Pretensiones curriculares y representaciones sociales de actores educativos en el programa de Enfermería de la Universidad Francisco de Paula Santander - Cúcuta, Colombia
}

\author{
Formative research: Curriculum pretensions and social representations of educational actors in the Nursing \\ program at the University of Francisco de Paula Santander in Cucuta, Colombia
}

Audin Aloiso Gamboa-Suárez1* orcid.org/0000-0001-9755-6408

Ruby Vargas-Toloza' orcid.org/0000-0002-3929-091X

César Augusto Hernández-Suárez¹ orcid.org/0000-0002-7974-5560

1 Universidad Francisco de Paula Santander. Cúcuta, Colombia

Fecha de recepción: Junio 9 - 2016

Fecha de revisión: Noviembre 11 - 2016

Fecha de aceptación: Marzo 24 - 2017

Gamboa-Suárez AA, Vargas-Toloza R, Hernández-Suárez CA. Investigación formativa: Pretensiones curriculares y representaciones sociales de actores educativos en el programa de Enfermería de la Universidad Francisco de Paula Santander - Cúcuta, Colombia. Univ. Salud. 2017;19(1):60-66. DOI: http://dx.doi.org/10.22267/rus.171901.69

\begin{abstract}
Resumen
Objetivo: Analizar los principales discursos sobre investigación formativa presentes en las políticas educativas y las representaciones sociales de actores educativos en el programa académico de Enfermería de la Universidad Francisco de Paula Santander. Materiales y métodos: Las orientaciones metodológicas parten del análisis del discurso vertical cualitativo para lograr comprender los enunciados de los documentos institucionales y el grupo focal para identificar las representaciones sociales de los autores. Resultados: El estudio logra develar que la investigación formativa es sistemática durante todo el programa académico de Enfermería, tangencialmente evidenciado en las pretensiones curriculares donde la formación en los paradigmas de investigación fortalece tanto la teoría como la práctica. Conclusiones: El estudio muestra una relación significativa entre los enunciados de las políticas educativas del programa y los relatos de los sujetos objeto de estudio.
\end{abstract}

Palabras clave: Curriculum; investigación en educación de enfermería; escuelas de enfermería. (Fuente: DeCS, Bireme).

\begin{abstract}
Objective: To analyze the main speeches on formative research found in educational policies and the social representations of educational actors in the academic program of Nursing at the University of Francisco de Paula Santander. Materials and methods: The methodological guidelines start from the analysis of the qualitative vertical discourse to understand the statements of the institutional documents and the focus group to identify the social representations of the authors. Results: The study revealed that formative research is systematic throughout the academic program of Nursing, which is also tangentially evidenced in the curriculum pretensions, where the formation in the paradigms of research strengthens both the theory and practice. Conclusions: The study shows a significant relationship between the statements of the educational policies of the program and the stories of the individuals under study.
\end{abstract}

Keywords: Curriculum; research in nursing education; schools of nursing. (Source: DeCS, Bireme). 


\section{Introducción}

La investigación formativa es un tema que ha interesado a investigadores de todos los ámbitos de la educación superior ${ }^{1-5}$. Su importancia en los procesos de formación de los estudiantes como base para la conformación de semilleros de investigación y la construcción de conocimiento en las instituciones educativas genera una especial atención, no sólo en indagar por los procesos de formación profesional sino para cumplir con una de las misiones fundamentales de la universidad 6 .

Estudios como el de Parra ${ }^{1}$, han revelado que la investigación formativa es un rasgo esencial y definitorio de la identidad de la universidad contemporánea. Restrepo $^{7}$ señala que la investigación formativa en el ámbito del pregrado se refiere a una manera de aprender (formar en) la lógica y las actividades propias de la investigación científica.

Cortés et al. ${ }^{8}$, afirman que la investigación formativa es un espacio de formación de investigadores que, mediante el desarrollo de un conjunto de actividades relacionadas con la investigación, dotan a los estudiantes de todos los niveles de habilidades necesarias para la producción académica. Este estudio fomenta una reflexión importante de la relación entre docencia e investigación y la relación entre la formación para la investigación y la misión investigativa de la educación superior, pasa por la precisión en torno a la investigación formativa y a la investigación científica en sentido estricto, más ligada la primera al pregrado y a la especialización, y la otra más propia de la maestría y el doctorado y de la materialización de la misión investigativa de la Universidad.

Para Gamboa algunos trabajos evidencian que la formación en investigación que reciben los estudiantes en los niveles de pregrado se considera como un requisito más y no se propone un efectivo proceso educativo que genere en los alumnos un verdadero interés por la pregunta y por develar el porqué de los fenómenos ${ }^{9}$. En este sentido, el mismo autor señala que los estudiantes deben comprender que una investigación científica no implica un tortuoso camino de incertidumbre, ensayos y errores de protocolo y mucho menos como un requisito para obtener un título universitario, sea cual sea el nivel educativo.

La formación inicial en investigación es una lucha por la sensibilización y la pasión por la construcción del conocimiento, es en cierto sentido rendir tributo a nuestro científico favorito, es el amor por la transformación del mundo, de una pequeña porción de nuestra sociedad o incluso, por nuestro salón de clase ${ }^{6}$.

Por estos argumentos este estudio pretendió realizar una valoración cualitativa a las políticas educativas que orientan los procesos de formación investigativa en el programa académico de Enfermería de la Universidad Francisco de Paula Santander (Cúcuta Colombia) e interpretar los relatos de los estudiantes y docentes del mismo programa académico.

\section{Materiales y métodos}

El estudio asume un enfoque cualitativo, puesto que como lo afirman Muñoz, et al.10, pretende identificar la naturaleza profunda de las realidades y su estructura dinámica, que en este caso son los discursos de los sujetos sobre la investigación formativa y los significados que hacen parte de los enunciados de los documentos institucionales.

El horizonte metodológico que guió este estudio fue de orden hermenéutico que confirió al investigador herramientas de análisis para descifrar lo que dicen los datos recolectados. La hermenéutica como método de investigación trata de comprender los textos, (tanto los presentes en los documentos del programa académico de Enfermería, como los que surgen de los relatos de docentes y estudiantes); comprenderlos en este sentido es ubicarlos en sus contextos respectivos, con esto el investigador los entiende y analiza frente a sus autores y contenidos ${ }^{11}$. Según Martínez ${ }^{12}$, comprender mejor un autor, más de lo que él mismo se entiende. 
Según Koselleck et al.13, la hermenéutica implica una serie de interpretaciones que se expresan lingüísticamente, partiendo de las categorías que emergen de los discursos de los actores. Plantean que la posibilidad de la interpretación que se da en el contexto del diálogo entre un horizonte de entendimiento y el mundo vital trasciende a los referentes de espacio y tiempo. Esto se traduce en adentrase en la conciencia discursiva de los sujetos para lograr extraer sus relatos e interpretar sus diferentes posturas representacionales ${ }^{14}$.

\section{Técnicas de recolección}

La técnica principal para el análisis de los datos fue el análisis de contenido, el cual consiste en "una recopilación de información que permite estudiar el contenido manifiesto de una comunicación, clasificando sus diferentes partes conforme a categorías establecidas por el investigador, con el fin de identificar de manera sistemática y objetiva dichas categorías dentro del mensaje"15. El corpus documental que hizo parte de este análisis, hace referencia al documento de Acreditación y el Proyecto Educativo del programa académico de Enfermería de la Universidad Francisco de Paula Santander.

Otra técnica de recolección fue el grupo focal, forma de interacción colectiva entre el investigador y un número determinado de sujetos en un espacio común, favoreció la recolección de datos sobre la construcción colectiva de significados, que en este caso específico hace referencia a la investigación formativa en los procesos de enseñanza aprendizaje. Para aplicar esta técnica participaron 18 estudiantes de últimos semestres del programa de Enfermería y 12 profesores. En criterio de muestreo para la selección de los participantes, se impuso la muestra intencional donde se priorizó la profundidad sobre la extensión y de esta manera se redujo su amplitud numérica ${ }^{12}$. Es decir, que el criterio de muestreo fue la selección de estudiantes que habían cursado las asignaturas de investigación y docentes que orientan o han orientado dichas asignaturas. En los hallazgos y discusión se muestran los relatos con códigos de la siguiente manera: D4 (Relato del docente 4) y E16 (Relato del estudiante 16). Estas descripciones se tomaron teniendo en cuenta la relevancia del enunciado para la categorización y posterior interpretación.

En la Tabla 1 y la Figura 1 se resume el proceso de análisis e interpretación de los resultados:

Tabla 1. Proceso de análisis documental

\begin{tabular}{|c|c|}
\hline Fase & Argumento \\
\hline $\begin{array}{l}\text { Selección de la } \\
\text { comunicación } \\
\text { que será } \\
\text { estudiada }\end{array}$ & $\begin{array}{l}\text { Definición del protocolo de análisis y tipo } \\
\text { de análisis (Descriptivo: para la } \\
\text { identificación y catalogación de la } \\
\text { realidad empírica de los textos, también } \\
\text { llamado análisis documental) }\end{array}$ \\
\hline $\begin{array}{l}\text { Selección de } \\
\text { las categorías }\end{array}$ & $\begin{array}{l}\text { 1)Identificación de fuentes primarias } \\
\text { (Documentos normativos de rigor de la } \\
\text { institución) } \\
\text { 2) Delimitación de las macrocategorías y } \\
\text { subcategorías }\end{array}$ \\
\hline $\begin{array}{l}\text { Diseño del } \\
\text { libro de } \\
\text { códigos }\end{array}$ & $\begin{array}{l}\text { 1) Delimitar la naturaleza del corpus: qué } \\
\text { contiene, para qué sirve, cómo } \\
\text { interpretarlo } \\
\text { 2) Identificar las unidades de análisis: } \\
\text { formas de segmentar el corpus } \\
\text { 3) Codificación de las fichas de análisis }\end{array}$ \\
\hline & $\begin{array}{l}\text { 4) Ordenar y archivar las fichas de } \\
\text { análisis }\end{array}$ \\
\hline Interpretación & $\begin{array}{l}\text { Interpretar los resultados: triangulación } \\
\text { de los datos con la teoría formal }\end{array}$ \\
\hline
\end{tabular}

Fuente: Gamboa Suarez et al. ${ }^{16}$
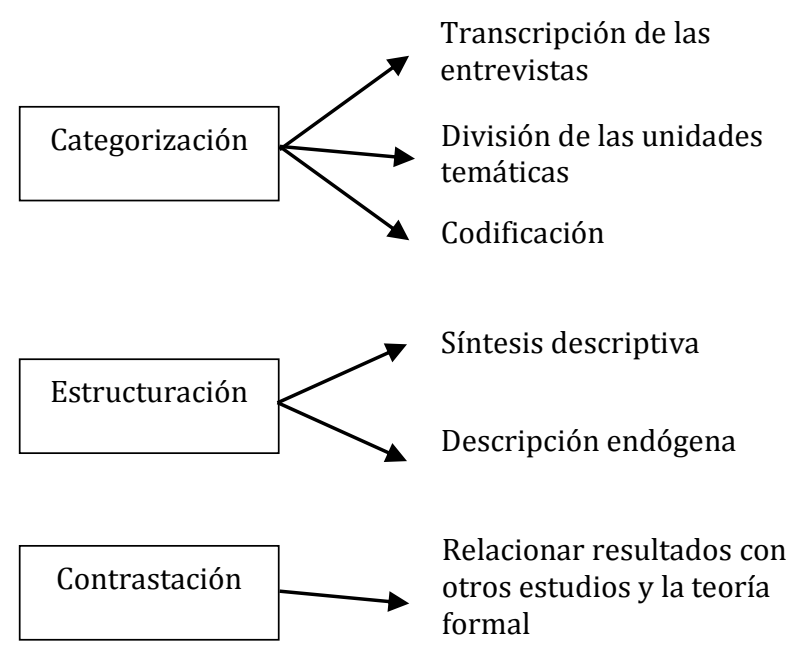

Figura 1. Análisis e interpretación de los relatos Fuente: Martínez ${ }^{12}$. 
El análisis de resultados se realizó con apoyo del software Atlas.TI 7.5.2, logrando así la división de contenidos en unidades hermenéuticas efectuando simultáneamente triangulación de fuentes documentales. Posteriormente, se procedió a la clasificación de la información existente en las unidades hermenéuticas con relación a las subcategorías y de esta manera iniciar con la interpretación teórica ${ }^{17}$, la cual se evidencia en los resultados desde la triangulación entre la teoría formal, los datos cualitativos recolectados y la interpretación del investigador.

\section{Resultados y discusión}

\section{Experiencia curricular y actitud científica}

Los discursos presentes en los documentos del programa académico de Enfermería dan cuenta de la investigación formativa como el conjunto de procesos que permite desarrollar y mantener una actitud de indagación, que enriquecida con teorías y modelos investigativos, permite la reflexión de la práctica profesional y el avance del conocimiento ${ }^{18}$. De este modo, según estos enunciados, el estudiante no sólo debe estar en contacto con los saberes ineludibles sino con procesos de investigación que le permitan analizar contextos y reconocer estrategias de indagación sistemática, apropiando los valores de la investigación, es decir, conocimiento del campo de trabajo, continuidad, crítica, rigor y compromiso con el problema ${ }^{18}$.

Por otra parte, advierten los discursos del programa académico de Enfermería que las estrategias pedagógicas para la investigación formativa se plasman en la práctica cuando el estudiante se prepara inicialmente en las metodologías y técnicas de investigación aplicada, donde el estudiante se apropia, identifica y reconstruye los conceptos fundamentales del método científico y de los procesos de investigación, luego se integra a un grupo de investigación en donde acompaña a sus miembros en el proceso ${ }^{18}$.

Interpretando estos discursos institucionales sin duda, la investigación formativa se comprende como el desarrollo de una actitud, el dominio de un método y el ejercicio de una práctica. El desarrollo de una actitud investigativa es posible de lograr a través de las experiencias curriculares con la ejercitación intensa de la capacidad racional y lógica de la mente del estudiante, al momento de abordar los hechos científicos y sociales de manera activa mediante el desarrollo de las competencias de análisis y crítica y la capacidad de formular preguntas alrededor de los fenómenos en estudio antes que la recepción pasiva de las respuestas sobre los mismos, característica de la enseñanza tradicional ${ }^{19}$. Igualmente, la conciencia de que la exploración de los límites del conocimiento actual representa un desafío y el reconocimiento de que la búsqueda de la verdad es una de las motivaciones fundamentales de la humanidad.

La actitud investigativa según Muñoz et al.20, forma parte intrínseca del comportamiento del investigador puesto que se configura como una disposición individual para resolver de manera crítica las diferentes circunstancias de incertidumbre, racionalidad y complejidad propia del trabajo científico; estas actitudes se adquieren en experiencias socializadoras y de servicio ofrecidas en los espacios de formación, además, permitirá abordar la descripción y análisis de cualquier problema en el campo disciplinar con rigor científico, dentro de las competencias propias de los programas académicos.

Las experiencias curriculares deberán permitir igualmente el dominio paulatino del método científico, es decir, la habilidad para reconocer el problema de investigación, formular las preguntas pertinentes y decidir el enfoque y paradigma investigativo que más se ajusta a su naturaleza. El currículo, por lo tanto, debe ofrecer las opciones necesarias para que el estudiante pueda decidir entre concepciones, teorías, métodos, procesos e instrumentos de investigación en un marco filosófico específico sobre la naturaleza de la ciencia y su función social21. Por lo tanto, Es indispensable el desarrollo de las competencias específicas por parte de los docentes, tanto en el campo epistemológico, como metodológico de las disciplinas que orientan. 
Se logra evidenciar que las opiniones de los docentes del programa de Enfermería no distancian de estos discursos, pues manifiestan que la investigación formativa radica en "ofrecer las herramientas metodológicas para que el estudiante pueda indagar, cuestionarse y tener un horizonte en sus aspiraciones investigativas y estas se plasmen en su trabajo de grado" (D4).

Los estudiantes manifiestan que: "la investigación formativa se plasma en la práctica cuando vemos investigación cualitativa $\mathrm{y}$ cuantitativa, y hay que hacer un proyecto con las dos metodologías. En cuantitativa puede ser en el quirófano, en la parte ambulatoria, para mirar lo que era la parte de incidencia de infección en las post cirugías y la cualitativa, cuando se observa el contexto cultural de la persona en cuanto al cuidado que se le daba al paciente desde lo subjetivo y de este modo generar un conocimiento profundo y no simplemente un conocimiento superficial"(E12).

Importantes afirmaciones que demuestran que la formación en investigación está consolidando una cultura investigativa y formando en el rigor metodológico de los dos paradigmas tanto cuantitativo como cualitativo, que posiblemente favorecerá sus pretensiones investigativas para obtener el título y en su ejercicio profesional.

Estos resultados se pueden contrastar con el estudio de Molina et al. ${ }^{22}$, quienes se propusieron identificar la apreciación del estudiante de medicina sobre la formación que recibe por parte de su universidad y el nivel que posee sobre el proceso de investigación, los resultados demuestran una tendencia favorable de los estudiantes a la capacitación por parte de la universidad en cuanto a metodología de la investigación y búsqueda de información. Es decir, que la universidad debe garantizar no sólo la investigación formativa sino también la más avanzada en un proceso de maduración y conjugarlas de acuerdo a las necesidades de aprendizaje $^{23}$ y a las necesidades sociales del contexto.

Según estos enunciados la actitud científica y el dominio metodológico se concretan en la práctica investigativa concebida como una actividad que intersecta todas las experiencias curriculares. Esta práctica entendida como ejercicio integrador presenta diferentes alternativas en el campo del currículo de los programas como actividades de investigación guiada en seminarios investigativos y en los tópicos generativos e hilos conductores ${ }^{24}$.

\section{Consumo de conocimiento y desarrollo del pensamiento crítico}

Otro hallazgo relevante relacionado con la investigación formativa es el enunciado que se muestra en los documentos institucionales, donde se representa la investigación como consumo de conocimiento, elemento necesario para ejercer la práctica formativa en los diferentes escenarios donde el estudiante interactúa ${ }^{18}$. El llamado consumo de conocimiento es fundamental en los procesos de formación del estudiante de Enfermería, pues estos deben tener un referente desde la investigación científica que soporte su práctica de cuidado del otro. Este es un rasgo importante según estos discursos, pues la actualización en el campo médico y en el campo de la enfermería es fundamental para que las prácticas de los estudiantes tengan un rigor científico y a su vez sean prácticas no instrumentalizadas sino reflexionadas a la luz de la teoría y la ciencia.

$\mathrm{Al}$ respecto los docentes manifiestan que: "consumir conocimiento es como alimentarse de la teoría, de que es lo que sirve y que es lo que se debe mejorar, que es lo que se debe aprender de buena manera, es como decir una dieta saludable, uno tiene que tratar de llevar al aula o a la práctica, todas las temáticas, las ideas, los problemas que se puedan resolver y que sirvan para enriquecer el desempeño de cada individuo, de cada estudiante, de cada profesora" (D3).

De igual modo, los estudiantes afirman que: "el consumo de conocimiento es un recurso muy importante porque da las últimas pautas de cuidado del paciente con investigaciones aceptadas por la ciencia, y en este sentido uno puede analizar si este nuevo conocimiento es pertinente o no para los casos que estamos estudiando" (E16). 
Las anteriores afirmaciones confieren a la investigación formativa el desarrollo del pensamiento crítico y científico en los estudiantes. En términos de Restrepo ${ }^{25}$ juega un papel fundamental en los procesos de enseñanza aprendizaje y se convierte en un "tema-problema pedagógico", que se refiere en concreto al papel que debe cumplir la investigación en el aprendizaje de habilidades para brindar los insumos para lo que será en el futuro la investigación en sentido estricto, es decir, más que construir conocimiento con un método riguroso y sistemático, abordar un proceso de aprendizaje y de transformación del ser humano y de este modo comprender que la educación es una forma de intervención en el mundo que implica libertad y autoridad para una toma consciente de decisiones mediadas por el saber escuchar y el reconocimiento de otras posturas teóricas y epistemológicas ${ }^{26}$.

El consumo de conocimiento también implica un avanzado manejo de la tecnología, técnicas de rastreo de la información en bases de datos especializadas y la utilización de software para análisis de la información. Estas competencias según Rojas-Betancur et al.27, deben ser desarrolladas de manera eficaz tanto docentes como estudiantes y de esta manera asumir los retos pedagógicos y didácticos para la enseñanza de la investigación en la universidad.

\section{Conclusiones}

El estudio logra develar que la investigación formativa es sistemática durante todo el programa académico de Enfermería de la Universidad Francisco de Paula Santander (Cucuta), tangencialmente evidenciado en las pretensiones curriculares donde la formación en los paradigmas de investigación está presente en los diferentes semestres tanto en la teoría como en la práctica.

Por otra parte, se da un valor preponderante a lo que el programa llama "consumo de conocimiento", término relacionado a la búsqueda de investigaciones actuales sobre los últimos avances en el campo de la enfermería para aplicarlos en sus diferentes escenarios de práctica.

La experiencia investigativa plasmada y puesta en práctica en el programa objeto de estudio presenta una posibilidad de relaciones entre los estudiantes y los docentes, da lugar a espacios de interacción, confrontación y asociación que enriquecen los resultados individuales $\mathrm{y}$ permiten introducir cambios en las prácticas como producto de la construcción permanente del conocimiento que propicia la práctica investigativa.

Se hace imperativo seguir fortaleciendo el vínculo entre investigación y la formación como se demuestra en el estudio y de esta manera asegurar que la educación cumpla la función social para la cual fue creada. Esto implica que los profesores se comprometan con la investigación en sus campos de trabajo como en el aula, para así formar individuos que, a su vez, puedan continuar construyendo saber más allá de las fronteras de la Escuela.

\section{Financiación}

Estudio financiado por el fondo de investigación de la Universidad Francisco de Paula Santander (FINU) según contrato No. 035-2015 y apoyado por el Grupo de Investigación en Estudios Sociales y Pedagogía para la Paz (GIESPPAZ Categoría A en Colciencias).

Conflicto de intereses: Ninguno declarado.

\section{Referencias}

1. Parra C. Apuntes sobre la investigación formativa. Educ y Educ. 2004;7:57-77.

2. González Agudelo EM. La investigación formativa como una posibilidad para articular las funciones universitarias de la investigación, la extensión y la docencia. Rev Educ y Pedagog. 2006;XVIII:101-10.

3. Lozano Casabianca GA, Ochoa Hoyos AM, Restrepo Mesa SL. La articulación entre investigación, docencia y extensión en un programa universitario de Nutrición y Dietética. Perspect en Nutr Humana. 2012;14(1):7183.

4. Bolívar Osorio RM. Los modos de existencia de la estrategia de semilleros en Colombia como expresiones de la comprensión de la relación entre investigación formativa y la investigación en sentido estricto. 
Múltiples lecturas, diversas prácticas. Agora USB. 2013;13(2):433-41.

5. Zarate Cohecha B, Villalba Gómez JA, Ruiz Mendoza LO. Revista Digital Academia y Virtualidad. Acad y Virtualidad. 2013;6(1):43-52.

6. Gamboa Suárez AA. Investigación formativa en los programas de pregrado: comprensión de los discursos institucionales $y$ de actores educativos en una universidad colombiana. In: Congreso Internacional de Educación. 2016. p. 1835-47.

7. Restrepo B. Investigación formativa e investigación productiva de conocimiento en la universidad. Nómadas (Colombia). 2003;18(1):195-202.

8. Cortés Nieto J del P, Londoño Toro B, Luna de Aliaga BE, Palacios Sanabria MT, Torres Villarreal ML. Investigación Formativa y nuevas propuestas pedagógicas en las Facultades de Derecho. Caso: Facultad de Jurisprudencia de la Universidad del Rosario. Studiositas. 2008;3(1):28-33.

9. Gamboa Suárez AA. Diagnóstico sobre la incidencia de los cursos de profundización en la Universidad Francisco de Paula Santander, como modalidad proyecto de grado. Cúcuta; 2010.

10. Muñoz García PA, Gamboa Suárez AA, Urbina Cárdenas JE. Deberes ciudadanos y diversidad cultural: comprensión de los discursos de estudiantes y docentes desde la alteridad y la subjetividad. Infancias Imágenes. 2014;13(2):23-32.

11. Gamboa Suárez AA. Calidad de la educación superior. Pretensiones y realidades institucionales. Bogotá: ECOE Ediciones; 2016.

12. Martínez Miguelez M. Ciencia y arte en la investigación cualitativa. México: Trillas; 2006.

13. Koselleck R, Gadamer H-G. Historia y hermenéutica. Bracelona: Paidós; 1997.

14. Muñoz García PA, Gamboa Suárez AA, Montes Miranda AJ. Participación crítica y democrática: comprensión de los discursos de actores educativos Reviewed. Zo Próxima. 2015;22:56-68.

15. Ocampo Ramos BA, Páramo P. Análisis de contenido: un ejercicio explicativo. In: La investigación en ciencias sociales: técnicas de recolección de información. Bogotá: Universidad Piloto de Colombia; 2008.

16. Gamboa Suárez AA, Lago de Vergara D, Hernández Pina F. Calidad de la docencia universitaria comprensión de los discursos y políticas institucionales de una universidad pública en norte de Santander. Saber Cienc y Lib. 2016;11(1):197-210.

17. Muñoz García PA, Gamboa Suárez AA, Urbina Cárdenas JE. Educación Política: Una Mirada a la Educación Media. Bogotá: ECOE Ediciones; 2012.

18. Programa Académico de Enfermería CC. Informe de autoevaluación con fines de acreditación programa de Enfermería. Cúcuta; 2013.

19. Zubiría Samper J de. Modelos pedagógicos Hacia una pedagogía dialogante. 2a ed. Bogotá: Cooperativa Editorial Magisterio; 2006.

20. Muñoz Cabas D, Aular de Durán J, Reyes LM, Leal M. Actitud investigativa en estudiantes de pregrado: indicadores conductuales, cognitivos y afectivos. Multiciencias. 2010;10:254-8.
21. Stenhouse L. Investigación y desarrollo del curriculum. 5ta ed. Madrid: Ediciones Morata; 2003.

22. Molina-Ordóñez J, Huamaní C, Mayta-Tristán P. Revista Peruana de medicina experimental y salud pública. Rev Peru Med Exp Salud Publica. 2008;25(3):325-9.

23. De la Ossa VJ, Pérez CA, Patiño PR, Montes VD. La investigación formativa como una necesidad en el pregrado. Rev Colomb Cienc Anim - RECIA. 2012;4(1):1-3.

24. Blythe T. The teaching for understanding guide [Internet]. San Francisco: Jossey-Bass Publishers; 1998.

25. Restrepo Gómez B. Conceptos y Aplicaciones de la Investigación Formativa, y Criterios para Evaluar la Investigación científica en sentido estricto. Bogotá; 2011.

26. Muñoz García PA, Gamboa Suárez AA, Urbína Cárdenas JE. Formación ciudadana: discursos de educadores y estudiantes sobre las actitudes y el pensamiento crítico orientados al ejercicio de la ciudadanía. Rev Interam Investig Educ y Pedagog RIIEP. 2014;7(1):125-47.

27. Rojas-Betancur HM, Méndez-Villamizar R. Cómo enseñar a investigar. Un reto para la pedagogía universitaria. Educ y Educ. 2013;16(1):95-108. 